\title{
Overview of Important State-of-the-Art Technologies in Offshore Wind Energy Systems
}

\author{
Yuan-Kang $\mathrm{Wu}^{\mathrm{a} *}$, Li Wang ${ }^{\mathrm{b}}$, Yong-Qing Huang ${ }^{\mathrm{c}}$, Shu-Wei Liu ${ }^{\mathrm{c}}$ \\ ${ }^{a}$ National Chung-Cheng University, No.168 University Road, Minghsiung, Chaiyi 62102, Taiwan \\ ${ }^{b}$ National Chung-Kung University, No.1, University Road, Tainan City 701, Taiwan \\ ${ }^{c}$ Industrial Technology Research Institute, Green Energy and Environment Research Laboratories, Hsinchu, 31040, Taiwan
}

\begin{abstract}
Modern wind plants in offshore wind farms have grown in capacity into facilities that can no longer be ignored in transmission planning studies. Additionally, a large penetration of wind generation in an offshore wind farm into the power system will bring a number of challenges on system planning, operation, and impact analyses. This paper will provide an overview of the important state-of-the-art technologies in offshore wind energy systems, including the designs on internal electric system of an offshore wind farm, transmission system to shore, generator type, system frequency, transmission cable, communications medium and SCADA system, offshore substation and transformer. Additionally, the development on reactive power compensation, collection grid topologies, redundancy design for improving system reliability, power quality standard and measurement, and protection scheme on switching transient will also illustrated in this paper.
\end{abstract}

Keywords: Offshore wind farm, internal electric system, reactive power compensation, collection grid topology, redundancy design, power quality

\section{Introduction}

The interest in the utilization of offshore wind power is increasing significantly worldwide. The reason is that the wind speed offshore is potentially higher than onshore, which leads to a much higher power production. The offshore wind power potential can be considered to be significant. For Europe, the theoretical potential is estimated to lie between 2.8 and $3.2 \mathrm{PWh}$ and up to $8.5 \mathrm{PWh}{ }^{[1]}$. However, investment costs for offshore wind power are much higher than those for onshore installations. Until now, practical experience from offshore wind energy projects is limited. The installation and supporting structure of offshore wind turbines is much more expensive than that of onshore turbines.

By the end of 2011, global installed wind power capacity increased by $24.1 \%$ during the year, and now stands at $197.0 \mathrm{GW}^{[2]}$.In terms of cumulative capacity, the UK (1,341 MW) and Denmark (854 MW) remain the two biggest markets for offshore wind, followed by the Netherlands (247 MW), Belgium (195 MW) and Sweden (163 MW). A further ten offshore projects are currently under construction. When these are fully grid connected over the coming years, Europe's installed offshore capacity will rise to 6,133 $\mathrm{MW}^{[3-4]}$.

Large-scale offshore wind farms would bring many challenges to system planners and operators, manufacturers, and other participants on wind energy system. It is important to investigate the important issues on modern wind farm planning, operation, and impact analysis on system grid. Therefore, this paper will provide an overview of the important state-of-the-art technologies in offshore wind energy systems, including the designs on internal electric system of an offshore wind farm, transmission system

\footnotetext{
* Manuscript received July 7, 2012; revised August 18, 2012.

Corresponding author. Tel.: +886-5-2720411\#33232; E-mail address: allenwu@ccu.edu.tw.
} 
to shore, generator type, system frequency, transmission cable, communications medium and SCADA system, offshore substation and transformer. Additionally, the development on reactive power compensation, collection grid topologies, redundancy design for improving system reliability, power quality standard and measurement, and protection scheme on switching transient will also illustrated in this paper.

\section{Characteristic of Important Offshore Wind Farms in the World}

Table 1 shows the details for several important offshore wind farms in the world. They are installed in the UK, Denmark, and Germany. It is found that the average of water deep in these offshore wind farms is between $10 \mathrm{~m} \sim 20 \mathrm{~m}$; the average distance from shore is about $10 \mathrm{~km}$; the total install capacity ranges from 5MW to 504MW. As shown in Table 1, as the capacity of the wind farm is larger, then the distance from shore is longer; therefore the substation is required. As for the design of the voltage level in the transmission line, the voltage level exceeds $100 \mathrm{kV}$ if the wind farm capacity exceeds $110 \mathrm{MW}$; however, there are no rules if the wind farm capacity do not exceeds 110MW. Moreover, if the distance from shore exceeds $20 \mathrm{~km}$, then most of offshore wind farms in the world adopt the high-voltage cable (more than $100 \mathrm{kV})$.

Table 1. Important Offshore Wind Farms in the World

\begin{tabular}{|c|c|c|c|c|c|}
\hline $\begin{array}{l}\text { Offshore wind } \\
\text { farm project }\end{array}$ & $\begin{array}{l}\text { Water deep } \\
\quad(\mathbf{m})\end{array}$ & $\begin{array}{c}\text { Distance } \\
\text { from shore } \\
(\mathbf{k m})\end{array}$ & $\begin{array}{c}\text { Total install } \\
\text { capacity } \\
\text { (MW) }\end{array}$ & Wind turbine type & Substation \\
\hline Tunø Knob & 7 & 5.5 & 5 & Vestas V39-500kW & No \\
\hline Vindeby & 4 & 1.8 & 5 & Bonus 450kW & No \\
\hline Sprogo & 16 & 9.3 & 21 & Vestas-3.0MW & No \\
\hline Horns Rev 1 & 11 & 17.8 & 160 & Vestas V80-2MW & Yes \\
\hline Horns Rev 2 & 17 & 32.6 & 209 & Siemens SWP- 2.3MW & Yes \\
\hline Samso & 13 & 3.8 & 23 & Bonus 2.3 MW & No \\
\hline Rödsand 2 & 12 & 8.9 & 207 & Siemens SWP- 2.3MW & Yes \\
\hline Nysted & 9 & 10.7 & 106 & Siemens SWT-2.3MW & Yes \\
\hline Avedøre Holme & 2 & 0.4 & 11 & Siemens SWT-3.6MW & No \\
\hline Middelgrunden & 6 & 4.7 & 40 & Bonus 2MW & No \\
\hline Frederikshavn & 4 & 3.1 & 11 & $\begin{array}{l}\text { Nordex N90-2.3MW, Vestas } \\
\text { V90-3MW, Bonus 2.3MW }\end{array}$ & No \\
\hline Thanet & 23 & 17.7 & 300 & Vestas V90-3MW & Yes \\
\hline Greater Gabbard & 37 & 32.5 & 504 & Siemens SWT-3.6MW & Yes \\
\hline Kentish Flats & 5 & 9.8 & 90 & Vestas V90-3MW & No \\
\hline Lynn & 11 & 6.9 & 97 & Siemens SWT-3.6MW & No \\
\hline North Hoyle & 12 & 9.2 & 60 & Vestas V80-2MW & No \\
\hline Rhyl Flats & 11 & 10.7 & 90 & Siemens SWT-3.6MW & No \\
\hline Robin Rigg & 12 & 11.5 & 180 & Vestas V90-3MW & Yes \\
\hline Burbo Bank & 6 & 8 & 90 & Siemens SWT-3.6MW & No \\
\hline Barrow & 16 & 12.8 & 90 & Vestas V90-3MW & Yes \\
\hline Scroby Sands & 8 & 3.5 & 60 & Vestas V80-2MW & No \\
\hline Walney Phase 1 & 23 & 19.6 & 184 & Siemens SWT-3.6MW & Yes \\
\hline Gunfleet Sands I+II & 13 & 7.4 & 173 & Siemens SWT-3.6MW & Yes \\
\hline Blyth & 5 & 1.1 & 4 & Vestas V66-2MW & No \\
\hline $\begin{array}{c}\text { Beatrice } \\
\text { Demonstration }\end{array}$ & 45 & 24.2 & 10 & REpower 5MW & No \\
\hline Hooksiel & 5 & 3 & 5 & BARD 5MW & No \\
\hline $\begin{array}{l}\text { ENOVA Offshore } \\
\text { Project Ems } \\
\text { Emden }\end{array}$ & 2 & 0.6 & 5 & $\begin{array}{c}\text { Enercon E-112 } \\
4.5 \mathrm{MW}\end{array}$ & No \\
\hline Breitling & 0.5 & 0.1 & 3 & $\begin{array}{c}\text { Nordex N90 } \\
2.5 \mathrm{MW} \\
\end{array}$ & No \\
\hline Alpha Ventus & 30 & 56.2 & 60 & $\begin{array}{l}\text { Areva Multibrid 5MW, } \\
\text { REpower 5MW }\end{array}$ & Yes \\
\hline BARD Offshore 1 & 41 & 111.9 & 400 & BARD 5MW & Yes \\
\hline EnBW Baltic 1 & 19 & 17.1 & 48 & Siemens SWP- 2.3MW & No \\
\hline
\end{tabular}


In general, the electrical system of an offshore wind farm is composed of the following four parts: local collection system, integration system, transmission system, and grid interface ${ }^{[5]}$. The local collection system connects a number of wind turbines to a cluster using internal connecting cables. Collection systems are normally rated at medium voltage level. The maximum number of turbines per circuit is a function of the turbine rated capacity and the spacing of the turbines. Turbines in a large wind farm are therefore connected in a clustered arrangement, and these clusters are then integrated together by using the integration system; typically, integration systems raise the voltage level from MV to HV. The transmission system transfers the wind power to the external grid system at the point of common coupling (PCC). The voltage level of the transmission system could be MV or HV, which determines whether the offshore platform is required or not. If offshore platforms with transformers or converters (for AC/DC integrated networks) are required, there are various ways of integrating the wind turbine clusters together and connecting them to the transmission system.

The internal electric system of an offshore wind farm and its connection to the main power system pose new challenges. For large offshore wind farms with an AC network, higher voltage levels will certainly be useful in order to minimize power losses, but higher voltage levels may result in bigger transformers and higher costs for these transformers. In addition, the cost and size of switchgears will also increase with voltage levels.

A typical offshore wind farm may has hundreds of generators, which is outspread in the range of several to tens kilometers, so that, at a certain reliability level, there are many feasible schemes for the internal electric connection system in a wind farm. The planner must search for an optimal one from the feasible schemes, usually with the criterion of lowest cost. It should be noted that such optimization is of much significance. Cost of internal electric connection system is about $8 \%$ of total investment costs for an onshore wind farm, while the proportion is $18 \%$ for an offshore one ${ }^{[6]}$. Electric connection of the famous Horns Rev 150 MW offshore wind farm is discussed in ${ }^{[7]}$, and voltage and feeder pattern is determined from comparison among various feasible schemes.

For the cabling to shore, either high-voltage alternating-current (HVAC) or high voltage direct-current (HVDC) connections could be used. For HVDC connections, there are two technical options: line commutated converter (LCC) based HVDC and voltage source converter (VSC) based HVDC technology ${ }^{[8]}$. An HVAC transmission system consists of an AC based collector system; perhaps an offshore transformer station including offshore reactive power compensation; three-core polyethylene insulation (XLPE) HVAC cables to shore, and, onshore static VAR compensators. With an increasing distance to shore, reactive power compensation will be required at both ends of the cable. The main disadvantage of HVAC solutions is when wind farm size and distance to shore increase, load losses will increase significantly. Also, an increase in the transmission voltage level will lead to larger and more expensive equipment as well as more expensive submarine cables. The advantage of an LCC HVDC solution is comparatively low losses. In addition, the higher transmission capacity of a single cable compared with HVAC transmission or VSC based transmission can be an advantage for very large offshore wind farms ${ }^{[9]}$. VSC based HVDC technology is gaining more and more attention. It is marketed by ABB under the name HVDC Light and by Siemens under the name HVDC Plus. This comparatively new technology has only become possible as a result of important advances in high-power electronics, such as IGBTs. A VSC based HVDC transmission system consists of an AC based collector system, an offshore substation with the relevant converters, DC cables, and an onshore converter station. Its advantages are that it does not require a strong offshore or onshore AC network; furthermore, its active and reactive power supply can be controlled independently. Other often-emphasized advantages of a VSC based HVDC solution is the capability of four-quadrant operation, the reduced number of filters required, black-start capability and the possibility of controlling a number of variables such as reactive power, apparent power, harmonics and flicker ${ }^{[10]}$. Although the VSC based HVDC link has many advantages over HVAC and other transmission solutions, most of the offshore wind farms in the world still adopt AC alternatives; this is because of the comparatively small size and the short distance between the shore and the existing wind farms. As the size of future wind farms and the distance to shore is likely to increase, this might change. 


\section{Novel Technologies in the components of Offshore Wind Farms}

By investigating all the operational offshore wind farms, the average offshore generator power ratings over the last 10 years and 5 years are 2.63MW and 2.89MW respectively. The most popular generator is doubly-fed induction generator (DFIG), which accounts for almost a half of the total installed number of offshore wind farms. Permanent magnet synchronous generator (PMSG) and DFIG are the most popular generators in the present market, and the fixed-speed wind turbines for offshore application are not offered at all. The generation efficiency is important for the wind turbine selection. Under low wind speed condition, the efficiency of low-speed EESG is good; while the efficiency of the medium-speed PMSG is good under high wind speed. It is noted that systems with high-speed PMSG, high-speed SCIG, and highspeed EESG offers low efficiency in whole wind speed range. Additionally, system with high-speed DFIG offers low efficiency in low wind speed, but comparable efficiency around rated operation point to system with low speed PMSG. This is due to the fact that low loss in converter compensates the threestage gearbox loss ${ }^{[11-12]}$.

The weight of a wind turbine is another important consideration. In order to reduce turbine weight, directly-driven offshore generators are getting popular. However, development of directly-driven offshore generators requires addressing electro-magnetic, insulation, electric system, thermal and mechanical problems. Two factors make design special: the first is generator size; the second is the saline atmosphere causing the need for special encapsulation and ventilation.

Several experts suggest the use of a lower AC frequency within the offshore wind farm. Frequencies lower than 50 or $60 \mathrm{~Hz}$ are currently used mainly in electrified railway systems. If an HVAC transmission solution is used, the low AC frequency could be applied in the internal wind farm network and for the transmission system to shore. A lower AC frequency would therefore allow a smaller gear ratio for wind turbines with a gearbox, or a reduction of pole numbers for wind turbines with direct-driven generators, both consequences resulting in lighter turbines that are thus likely to be cheaper. Additionally, a low AC frequency will also increase the transmission capacity of HVAC transmission links or the possible maximum transmission distance, as a capacity charging current is significantly reduced at lower frequency. However, the disadvantage of this concept is that the transformer size will increase significantly and therefore transformers will be more expensive.

In terms of the cable system, the major manufacturers in the world include ABB, NSW, Nexans, Prysmian, and NKT. The material of most cables belongs to the AC Cross-Linked Polyethylene (XLPE) cable. In order to limit the energy losses, the offshore wind farms have to be connected to substations at higher voltage levels, which brings about increased reactive power production from the cables when using conventional technology and consequently growth of the requirements for reactive power compensation. In the future, high temperature superconducting (HTS) cables would become a novel transmission solution. Using the HTS cables, fault levels would become much lower and the wind farm can be connected at MV directly to the substation. In this new concept expensive and bulky transformation platforms and reactive power compensation units could be saved. However, the cooling system of the HTS cable is not easy to design.

The communications medium and SCADA system is also necessary in offshore wind systems ${ }^{[13-14]}$. The SCADA system for wind farm monitoring and control will require a communication network between the wind turbines in the wind farm, and back to the shore. Candidate media for this are copper twisted pair (RS485), fibre optic - multi mode, fibre optic - single mode, and radio telemetry. The twisted pair copper cable using RS485 drivers is the cheap conventional medium for communications. However, the disadvantages of copper connections are that there is no inherent isolation between the machines and that the drivers are susceptible to transient voltages. Fibre optic cables offer high bandwidth communication over long distances. They also have the advantage of galvanic isolation to electrical noise. Two types of cable are available, single mode and multi-mode: for single mode cable, data rates of up to 2 $\mathrm{Gb} / \mathrm{s}$ are available over distances of up to $100 \mathrm{~km}$. With multi-mode cables, data rates of up to $100 \mathrm{Mb} / \mathrm{s}$ are achievable over distances of up to $6 \mathrm{~km}$. The disadvantages of fibre optic cable are that it is less robust 
than copper and that it needs special tooling for making connections.

As for the transformers in an offshore environment, the IEE offshore regulations do not allow oil-filled transformers, because of the risk of fire. Other liquid-filled transformers must have a bund to prevent discharges to sea. The GL rules prefer dry-type transformers, but do not forbid oil-filled units. Offshore wind farms built to date have used both liquid and dry-type cast-resin transformers. It is expected that cast-resin transformers will predominate in offshore wind farms. They are commonly used indoors on offshore platforms. Although the weight is similar, cast-resin transformers are typically narrower than liquid-filled, and so design for removal is simpler.

Different technical transmission solutions have widely divergent requirements regarding the size of the offshore substation. In general, the size of an AC offshore substation will be only about a third of the size of the corresponding HVDC solution, owing to the significant space required by the converter stations. For onshore HVDC converter stations, LCC based converter stations need considerably more space than do VSC based systems. Regarding VSC converter stations, a larger capacity demand will require multiple VSC converter stations.

\section{Reactive Power Compensation in Offshore Wind Farms}

AC cable lines are characterized by many times as large shunt capacitance compared to overhead lines. The resulting charging current will limit the remaining cable capacity for active power transfer, increase the active power losses and increase the voltage along the line due to the Ferranti effect. In order to reduce these negative effects in long HVAC cable lines, reactive power compensation may need to be installed not only at the ends of the line but also along the line. A 400MWoffshore wind farm with two $150 \mathrm{kV}, 120 \mathrm{~km}$, cable systems, for instance, will require 150MVAR compensation offshore as well as onshore. In the Horns Rev wind farm, a 75 MVAr compensating reactor was installed in the middle section of the wind farm cable connection. The entire $150 \mathrm{kV}$ wind farm connection, including offshore transformer, sea cable, compensating reactor, land cable, onshore connection point and 167.5/410 kV autotransformer belong to the Danish Transmission System Operator. Therefore, the loading of an openend cable line due to charging current for various distributions of the reactive power compensation should be studied. These analyses include where to install reactive power compensators; i.e. the compensation is located at both ends, distributed equally along the line, or located at the middle.

\section{Collection Grid Topologies in Offshore Wind Farms}

The investment costs for the electrical system, collection grid, platform, platform switchgear and subtransmission is in the range of $10-15 \%$ of the total investment costs of the offshore wind park. In general, the overall function of the wind farm collector system is to collect power from individual wind turbine and maximize the overall energy generation by taking into account the installation cost and performance. Electrical collector systems can be designed using different layout depending on the wind farm size and the desired level of collector reliability. These topologies include radial, single return, single-sided ring, double-sided ring, star, multiple rings and multi-hub ring. The most straightforward arrangement of the collector system in a wind farm is a radial design, in which a number of wind turbines are connected to a single cable feeder within a string. The maximum number of wind turbines on each string feeder is determined by the capacity of the generators and the maximum rating of the subsea cable within the string. The main advantage of this layout is the low cable costs and the simple control algorithms. However, the downside is the comparatively poor reliability, since cable or switchgear faults at the hub end of the radial cluster may prevent all downstream turbines from exporting for the duration of the fault. The doublesided ring layout interconnects the last wind turbine in one string to the last wind turbine in the next string. The redundant circuits are also considered to be in service during normal operation. Regardless of the benefits of no redundant cable, the power ratings of the cables are larger. The star design is another way to reduce cable ratings and to provide a high level of security for the entire wind farm. Voltage regulation 
along the cables between wind turbines is also likely to be better in this design. However, there are additional expenses in longer cable runs and some short sections of higher-rated cabling, but these expenses are not likely to be significant. The more complex switchgear requirement at the wind turbine is the major cost implication.

There are advantages and weak points for the above-mentioned configurations of the electrical collector systems. However, the basic designs for current offshore wind farms are still radial designs. For example, the radial design has been adopted for the 160 MW Horns Rev offshore wind farm in Denmark, and it has been proposed for many other offshore wind farms at the planning stage, such as the $640 \mathrm{MW}$ Krieger's Flak and the 420 MW Cape Wind offshore wind farms in Sweden and USA, respectively.

Based on available literature, in addition to power collection grid in the wind farm and grid connection configuration, several other aspects for wind generation's realistic reliability assessment and the optimal layout for wind farms are also clearly important. They include wake effects, turbulence, wind turbine technology, and offshore environment: wake effects reduce total wind farm output to a fraction of what would be obtained if each wind turbine stood alone. In available literature, this seems to be considered mainly in more detailed models and is included as an efficiency coefficient assumed equal to $90 \%-95 \%$ of the total wind farm production. As for turbulence, it may not only reduce the power output of the turbine, but also cause vibration and noise, and even shorten the life of the turbine. Under normal circumstances, the value of the turbulence intensity is about 0.05 to 0.2 . When the wind farm is built in complex terrain, for safe operation of the turbine, generally the turbulence intensity needs to be controlled with 0.2 as the upper limit.

The optimization of electric distribution system of an offshore wind farm includes such issues: number of main substations, capacity and site of each substation, topology of connection of wind power generators with substations, satisfying technical restrictions, and minimizing total cost ${ }^{[15]}$.

\section{Redundancy Design for Offshore Wind Farms}

In order to obtain higher system reliability, redundancy may be introduced to offshore wind farms. However, most electric networks of offshore wind farms that are currently under discussion have no or very little redundancy. The turbines of a wind farm may be connected to a 'radial' network, as for example in Horns Rev. A radial network design means that a number of turbines are connected to one feeder. The maximum number of turbines on each feeder is determined by the maximum rating of the cable. If the cable is damaged the whole feeder will be disconnected until the fault is repaired. Redundancy might be achieved by enabling connections between feeders. The last wind turbine in one feeder could be connected to the last turbine in the next feeder, for instance. During normal operation, this connection would be open. If there is a fault in one of these two feeders, the connection would be closed. In the redundancy design, it has to be taken into account that the number of wind turbines that may be connected to one feeder will be lower, as the maximum cable rating must be able to cope with twice as many turbines in case the fault is close to the first turbine within one feeder. To achieve the redundancy schemes, additional equipments like switch gears should be equipped on each feeder cables to detect fault location, isolate fault component and form ring connections.

\section{Switching Transient in Offshore Wind Farms}

Large cable systems, such as offshore collection grids, are capacitive in nature. In such systems, it is important to have appropriate breaker equipment in order to connect, disconnect or trip the cable system properly. If not, charging/discharging currents can cause. These switching over-voltages occur at a level, which stresses the collection grid equipment and can make the circuit breakers to pre- or re-strike. Generally, large over-voltages will occur due to resonance between feeder cable capacitance and the nacelle transformer saturation differential inductances. It is important to investigate how these long lengths of submarine cable will influence the transient over voltages. 


\section{Power Quality in Offshore Wind Farms}

The methods specified in IEC 61400-21 to assess the impact of large offshore wind farms on the local voltage quality should be studied. The study would include voltage range, flicker and harmonics. Additionally, IEC 61400-21 recommends that load-flow studies are conducted to assess the influence of wind turbines on the steady state voltages. In IEC 61400-21, the contribution of wind turbines to voltage fluctuations is divided into continuous operation and switching operation: the continuous operation contributes to flicker because the active power and reactive power fluctuate due to wind speed fluctuations. Switching operations related to generator cut-in, cut-out and switching between generator windings also contribute to flicker. A fast change from one power level to another causes flicker. In the Nysted offshore wind farm, power quality measurements have been performed on wind turbines. Three phase currents and voltages are measured at the wind turbines together with the wind speed. The offshore wind farm consists of 72 turbines each with rated power 2.3 MW. The wind turbines use directly connected induction generators and thyristor switched capacitors for reactive power compensation on the low voltage terminals. From the measurement results, the voltage increases with the wind speed. However, in some cases the reactive power consumed by the wind farm causes the voltage to decrease. The effective voltage resulting from these opposite impacts can be provided by standard load flow studies for different wind speeds. Additionally, there is a significant relation between wind speed and voltage flicker which shows that the main source of flicker is the wind turbines.

\section{Conclusions}

A typical offshore wind farm may has hundreds of generators, which is outspread in the range of several to tens kilometers, so that, at a certain reliability level, there are many feasible schemes for the internal electric connection system in a wind farm. As offshore wind farms become larger and are placed further from the shore, several issues will become key features. The objective of this paper is to give further insight to the designs on internal electric system of an offshore wind farm, transmission system to shore, generator type, system frequency, transmission cable, communications medium and SCADA system, offshore substation and transformer. Additionally, the development on reactive power compensation, collection grid topologies, redundancy design for improving system reliability, power quality standard and measurement, and protection scheme on switching transient also illustrated in this paper.

\section{Acknowledgements}

The authors gratefully acknowledge the financial support by National Science of Council (NSC) of Taiwan under Grant NSC 100-3113-P-006-014 and Bureau of Energy, Ministry of Economic Affairs, R.O.C. under the Project "Development of Key Control Technologies for Distributed Energy System"

\section{References}

[1] Leutz R, Ackermann T. Suzuki A, Akisawa A, Kashiwagi T. Technical offshore wind energy potentials around the globe, In: Proc. of 2001 European Wind Energy Conference, 2011:pp. 789-792.

[2] Global Wind Report, 2011, Global Wind Energy Council.

[3] Harris A. The winds of change -power offshore wind. Engineering \& Technology, 2010; 5(2):40-43.

[4] Holttinen H, Orths AG, Eriksen P, Hidalgo J, Estanqueiro A, Groome F, Coughlan Y, Neumann H, Lange B, Hulle F, Dudurych I. Currents of change. IEEE Power and Energy Magazine, 2011; 9(6):47-59.

[5] Liang J, Jing T, Gomis-Bellmunt O, Ekanayake J, Jenkins N. Operation and control of multiterminal HVDC transmission for offshore wind farms. IEEE Trans. on Power Delivery, 2011; 26(4):2596-2604.

[6] Fuglsang P, Thomsen K. Cost optimization of wind turbines for large scale off-shore wind farms. Report from Risoe National Laboratory, 1998. 
[7] Christiansen P, Jørgensen KK, Sørensen AG. Grid Connection and Remote Control for the Horns Rev 150 MW Offshore Wind Farm in Denmark. Tech Wise, 2001; 1-7

[8] Xu L, Andersen BR. Grid Connection of Large Offshore Wind Farms Using HVDC. New York: John Wiley \& Sons, Ltd., 2005.

[9] Ackermann T. Wind Power in Power Systems. New York: John Wiley \& Sons, Ltd, 2005.

[10] Kim CK, Sood VK, Jang GS, Lim SJ, Lee SJ. HVDC Transmission: Power Conversion Applications in Power Systems, Singapore: John Wiley \& Sons (Asia), 2009.

[11] Semken RS, Polikarpova M, Röyttä P, Alexandrova J, Pyrhönen J, Nerg J, Mikkola A, Backman J. Direct-drive permanent magnet generators for high-power wind turbines: benefits and limiting factors. IET Renewable Power Generation, 2012; 6(1): $1-8$.

[12] O'Sullivan DL, Lewis AW. Generator selection and comparative performance in offshore oscillating water column ocean wave energy converters. IEEE Trans. on Energy Conversion. 2011; 26(2):603-614.

[13] Pettener AL. SCADA and communication networks for large scale offshore wind power systems. In: Proc. of IET Conference on Renewable Power Generation, 2011:1-6.

[14] Liu X. Quality of optical channels in wireless SCADA for offshore wind farms. IEEE Trans. on Smart Grid, 2012; 3(1):225232.

[15] Gonzalez-Longatt FM, Wall P, Regulski P, Terzija V. Optimal electric network design for a large offshore wind farm based on a modified genetic algorithm approach. IEEE Systems Journal, 2012; 6(1):164-172. 